\title{
Fixed, liquid, fluid. Rethinking the digital design process through the ecosystem model.
}

\section{Bollini, Letizia}

Department of Psychology, University of Milano-Bicocca, Italy. letizia.bollini@unimib.it

\begin{abstract}
According to the visions and conceptualizations from philosophers to design thinkers such as Habermas, Maturana \& Varela or Levin, the design applied to digital artefacts, products and services - due to the convergence of media, deceives and technologies - is becoming even more a bio-sphere, or, with the words of Vernadskij, a Noosphere. The cultural shifting is represented both in the process side and in the approach to the whole design materials and outcomes. On one hand, the organizational structure is moving from an "industrial" approach characterized by a waterfall-process - organized in subsequent structured phases - to an iterative activity — that cycle among ideation, prototyping, testing assessing and redesign phases before to implement and release a project- to the agile and lean approach of the information-era in which the project itself persist constantly in a work-in-progress status -where updates have replaced new releases. On the other hand, the object of the project itself is deeply changing according to a vision of a digital ecosystem and consequently to the design approach that is moving from a fixed - a twodimensional page borrowed-model - to a liquid, then fluid solutions beside the divergences of media and devices and the convergence of user context and experience. Paraphrasing Maldonando we're moving from virtual to real, from intangible to tangible, from the web to intelligent environment, both digital and physical. In this hybrid space design plays its challenge to change process and purpose embracing both a traversal and a deep approach to single elements and the eco-system in its wide complexity. Nevertheless this transition implies design to face with the challenges of emerging and upcoming phenomena: the designer education -skills, competences, methods- in an hybrid context, the anthropological mutation brought up by the new generation of digital natives and finally the social impact and emotional implication of the confluence of virtual and real experience - mediated by technologies — that people live in their daily life.
\end{abstract}

Keywords: digital ecosystem design, fluid user interface design, user centered-design, mobile user experience design, agile design methods. 


\section{Converging phenomena and centrifugal forces}

The traditional interactions, which people could experience with the different communication channels, were naturally linear until a couple of decades ago. The user has started doing and finalizing the tasks always in the same media channel, choosing from time to time which one was more suitable according to a multi-channel services offers. This multi-channel approach has been overtaken by a cross-media as clarified by Rosati (2011) in a post related to pervasive architecture.

The participative evolution of users' interactions enabled by the web 2.0 , the wide diffusion of social networks, the digital mutation of communication mass-media, sets the scenario in which happens a cultural migration defined by Jenkins (2006) as convergent culture or trans-mediality. That means a flow of contents coming from different platforms, cooperation among may different sectors of the media industry and the migration of the audience looking for new entertaining experiences.

However, Jenkins perspective lacks on media according of the conceptualization of media as a tool: "Medium can be seen according to an ambivalent point of view. On one hand it could be a tool on the other a way/manner. Computers themselves are 'meta-medium able to simulate others, but their essential is also the universality the ability of simulation' (Polillo, 1993). According to this idea, the computer extensively the digital support and languages - is a medium, but rather a tool that mean the designer operating field who designs the modalities of access and interaction between the user and information." (Bollini, 2004: 60).

The debuts of the first Phone presented in 2007 broke the digital scenario, at that time monolithic - based on web predominance. The revolution of an integrated and mobile experience based on cloud-services introduces many variables impacting on the design process, framework and complexities. Communication becomes not only cross-channels but multi-devices too. People initiate a task using a specific device in a coherent context - for example in the subway during commuting time going from home to workplacethen they prosecute the stared process using another device — when arrived in the office with a desktop computer. And finally - at the end of the day, lying relaxed on a sofa, watching TV — they finalize the order or control the status using a tablet: a totally different context compared to the one in which they began the process.

The experiential scenario could be so over-crowded of devices, interactions and activities acted at the same time, that we could talk about a systemic-environmental-multitasking to underline not only the simultaneity of the actions but also the social and structural contextually of the experience.

The user is no more asked to choose the most suitable device to complete a task, rather he/she access, disconnects, starts over the process from the last completed step, although in another context. The interaction moves therefor - also as a design focus - from the interface space to the different accesspoints, which — if transparent - allows the user to go in and out from different context and devices without perceiving any discontinuity.

The concept of transparency firstly introduced by Gui Bonsiepe (1993) to define one of the highest qualities of the Graphical User Interfaces, now is extended to the whole experiential system as a synonymous of the lack of friction cognitive.

Design, too, should be accountable for this activities and contents migration in a segmented and multifaceted scenario of interactive tools becoming the unifying factor for the user experience. The process itself should face, at its first beginning, the need to integrate, consolidate and decline the communication issues, the uses of information and services in the different users' case and scenarios, social, and media situation. 


\section{The digital ecosystem}

Already in the crossroad from paper and traditional communication media - off-line - designers have been manage a radical, conceptual change of consolidated paradigms and praxis, layered and established in the secular history of writing, publishing and advertising, that means in the culture of the dimensional mise-en-scene.

The mutant format, compared to the fixed measures of physical supports, the weak control and the poor expressive possibilities given by screen-typography, the many different and crazy displays results although the same design and front end development sources - limited by platforms' standards, browsers releases; the low quality of the images and the poor resolution of the displays and saving-formats —only bitmaps that means lossy - are just some of the critical transitions that designer have been facing the first transition from paper to web - according to Zelman work (2001) - now become standards them selves to plan and project a web interface and site.

The introduction of mobile devices, smartphone in 2007 and tablet in 2010, the family of tool to access the web, Internet services has become wider and differentiated increasing user scenarios, the social interactive flow along the everyday life time.

The design challenge is doubled: on one hand, design must face and solve the parcelling of interaction environments; on the other hand it should be able to unify the user experience.

According with the state of the art, the cultural debate too, is no more discussing of the project of a single communication artefact - the web site, the mobile app and so on — but rather of digital eco-systems. That means an interconnected system, in which single elements that are part of, interact among themselves and with the environmental context.

The naturalist metaphor applied to the phenomenological digital world seems to refer to the Noosphere conceptualization firstly introduced by Verdnaskij (1926) as the highest form of evolution of the biosphere and the human intelligent system in which some authors have already identified the collective intelligence then enabled by the Internet. The promising outcome of this comparison between technologies and the natural environment gives both a systemic vision of the phenomena coming also from the idea brought up by Maturana and Varela in their research work Tree of Knowledge: Biological Roots of Human Understanding (1984) and then adopted by Maldonano; and a gestaltic the approach whereby the whole is more than the sum of its single parts.

As underlined by Levin, the design issues is not to duplicate the same experience -often in a simpler manner - in all the devices enabling a "everything, everywhere, anytime" access, but rather to dynamically catch the user needs and context associated with the cross-migration from a device to another. The challenge is to focus on "right thing at the right place at the right time" in a context-driven perspective (Levine, 2014: 3) shifting to a new method which pillars are the "3Cs framework: consistent, continuous, and complementary". The focus moves from technology, to people, the devices that they own, in this moment, in this context to execute a specific task.

\section{From waterfall process to spiral model}

The design process has been deeply influenced by this shifting from sectorial —industrial, product, graphic, multimedia, web and so on- approach to an eco-systemic perspective. 
The referring point is no more a matter of scale - as stated by Rogers from spoon to the town - but rather a matter of focus, moving from the object both physical and virtual and its productive process to people and the experience that they would do of it in a user scenario to satisfy their explicitly and implicit needs.

The turning points are two and they referee to different level of design culture: the first pertains the subject of the design process and its out put - a unit, and artefact related to the whole ecosystemic world of the user and his/her needs in a specific context as previously described in tis paper- the second is a consequence of it. That means how the design process itself is redefined at its core to give answer to this new requirements in a progressively, accelerated and on-going changing of requirements and technological scenarios.

The project praxis was originally borrowed by the Tayloristic assembly-line model also due to the strong connection between design contribution and production process, just to mention the original label give to the discipline of industrial design according to the critical and historical evolution well synthetized by Maldonado in Disegno industriale un riesame in 1976. The design work was organized according to the so-called waterfall process. The whole project was structured along a linear sequence of phases, starting with analysis and requirements, going through concept, design, development and release. Each phase have a starting and ending date and specific documentation expecting to be transmitted to the next team. Often competences too are segmented in homogenous working group each one focalized on a limited part of the project itself and rarely able or involved from the beginning to contribute to the big picture.

Although this is a generalization of the waterfall process it is not so far from is more orthodox application.

This kind of approach implies a well-fixed goals; stable in time; expected out put and a very strong time management able to face with the unavoidable delay, change of course and entropic phenomena. Moreover it is only applied to project which outcomes have a significantly long-term life cycle due to the time-consuming effort of the whole process; otherwise it risks to deliver irrelevant objects by the time the project is finished.

But in the last two decades the world of design — both material and digital — has been deeply impacted by new phenomena: a wide acceleration of technologies evolution; a raising and fall of artefactual and production issues and praxis; the pollution of electronic and digital devices; virtual and augmented reality; Internet of Things where products and internet are converging; mobile revolution; wearables; smart-whatever and intelligent environment; auto-productions, industrial mini-series; digital art and crafts; makers movement; fab-labs; 3D-printing; robotics and automation; just to mention the most relevant emerging trends. Under the pressure of such disruptive impact the fixed and structured approach to design process have been radically modified moving from the sequential flow to a more iterative loop.

The introduction of the user-centered design approach in the ' $90 \mathrm{~s}$ as an essential requirement in design field has forced to interrupt the linear step by step process to insert user testing and assessment activities and subsequent redesign and release circles. This kind of conceptual framework was firstly introduced by Lehman in IBM as The Programming Process: "The design process is [...] seeded by a formal definition of the system, which provides a first, executable, functional model. It is tested and further expanded through a sequence of models, that develop an increasing amount of function and an increasing amount of detail as to how that function is to be executed. Ultimately, the model becomes the system." (Lehman 1969).

In more recent times - 2001 - in the field of coders and software the core principles of the agile approach were defined and declared in the Agile Manifesto. This working method is developed and carried out by small teams which "that deliver real, working software at all times, get meaningful 
feedback from users as early as possible, and improve the product over time in iterative development cycles. Developing software in an agile way allows developers to rapidly respond to changing requirements. Agile developers believe that where uncertainty is high there is no such thing as a perfect plan, and the further ahead you plan, the more likely you are to be wrong." (Beck 2001)

The agile approach seems to fit perfectly the liquid state of the art of the design discipline in the crossroad of material, digital and virtual instances. It guarantees shorter time phases (one to two weeks) and smaller deliverables in a more controlled and verified design-testing-development cycles. At the end of every single project iteration features released — called story — have already been tested and assessed with users: "This means requirements can change quite frequently through development. Along the way, refactoring takes place from time to time in order to ensure that features fit together into a single cohesive application. [...] At times, it will be necessary to jump out of the iterative flow and examine the whole system for congruency. Do components fit? If not, why? As you work through these potential problems, keep in mind the scope of your iteration and deliverables." (Anderson 2011).

Although Scrum is specifically an iterative and incremental agile software development framework (Verheyen 2013) for managing product development, it suggests a further develop of design processes. It defines "a flexible, holistic product development strategy where a development team works as a unit to reach a common goal, challenges assumptions of the 'traditional, sequential approach' to product development, and enables teams to self-organize by encouraging physical co-location or close online collaboration of all team members, as well as daily face-to-face communication among all team members and disciplines in the project.” (Takeuchi \& Nonaka 1986).

\section{Releases vs. evolution}

So, if the traditional approach to digital design evolution was a constant and accessible development for the worst case scenario in terms of infrastructure, OS and browser versioning and compliancy with recent W3C standards, monitors dimensions and resolutions, in 2003 at SXSW Steve Champeon and Nick Finck gave a speech titled Inclusive Web Design For the Future presenting the concept of progressive enhancement a reversed approach in front of the previous graceful degradation. A basic mark-up document is created, geared towards the lowest common denominator of browser software functionality, and then the designer adds in functionality or enhancements to the presentation and behaviour of the page, using the news technologies (Gustafson, 2008)

The remarkable voices and advocate of web standards such as Jeffrey Zeldman, Andy Clark or Molly Holzschlag slightly move from an orthodox and retrospective position to the new approach of evolutionary process become nowadays the standard in design and development work.

As well exemplified by the Facebook strategy, the progressive enhancement has become the standard de facto in upgrading main software platform and their interactions with the people. Facebook definitely went over successive releases when approaching the mobile development. If the first adopted strategy of disruptive turning point in its evolution were caused as well by marketing or re-branding radical changing -as happened in 2005 with the complete redesign of the user interface and experience realized by Aaron Sittig, the first graphic designer hired in FB or the to develop the Facelift project (Bret, 2011) - the last evolution were smooth and progressive after a couple of years FB were becoming a platform and facing the mobile revolution.

The last discontinuity has been the introduction of the timeline loudly disclaimed long before it becomes the new standard visualization of the post streaming. Initially people were invited to adopt the new 
display model to familiarize with giving the opportunity to go back in the previous wall until the deadline announced. When FB went mobile - mobile access have widely overcome the other access models - the evolution of the interface and of the interactive patterns are frequent but always partial and progressive. Small innovations allow the users to slowly adopt changes almost without noticing them and reshaping and rearranging small parts of their mental models instead of the whole picture, once, with a strong and shocking effort.

\section{From real to virtual and back}

If in the late '90s the reflection about the impact of electronic and information technologies on artefacts was focused on the miniaturization and virtualization of objects, nowadays the tendencies are both convergent and divergent.

On one hand a large number of the specialized equipment — such as computers, phones, calculators, photo and video cameras, watches, TVs, walk-man, Hi-Fi etc.- are collapsing in few single, multifunction portable devices - the smartphone above all — on the other the world around us is becoming smart and intelligent, able to directly interact with us and our personal digital appendices.

Apparently miniaturization has a threshold limit - dimensions of components and physical ergonomic measures of our bodies - virtualization has not. Information technologies are still migrating from objects to the environment and moving from specialized tools to everyday objects: supermarket shelves, ephemeral exhibition and museums, cars, appliances and the domestic landscape, the bookshelves of libraries and the books - the reading machine become virtual both in its physical form and conceptual content.

Paraphrasing the Maldonado work about Real and virtual (Maldonado 1994) we can now moving back from virtual to real, but with the deep difference, that this dichotomy, on the contrary, is now recomposing. Digital is a layer covering and embedding itself inside the artefactual landscape making neutral object interactive and able to begin a dialog with us mediated by our personal devices in a transparent manner. An insight of this anthropological change is given by Sherry Turkle, who firstly investigated the perception, the psychological effects and the impact of real and virtual life. According to her research and critical work we can emphasize how digital technologies are "more than just a tool, but part of the everyday personal and psychological lives [...] Technology catalyzes changes not only in what we do but in how we think." (Turkle 1995)

The ecological and systemic approach, therefor, is not only a matter of devices, media or channels among which the user migrate and switch from time to time according to his/her needs, but has also a cognitive and social meaning. Personal interactions bot in the physical world and in the digital sphere leave meaningful traces and real experiences in the intra-psychological life of people giving rise, in turn, to an emotional eco-system.

\section{The cross-boundary flow of hybrid design}

According to these new issues raised in the design practices the discipline itself needs a theoretical reshape and an open debate on its boundaries and challenges. The project culture is asked to open its competences to other field such as Information, Communication and Digital Technologies, Computer Science, Software Development, Coding, mark-up and scripting - digital tech side- Cognitive Psychology and Ergonomy, Sociology, Ethnography and User Research Methods — humanities and social 
science side - Information Architecture, user-centered and co-design and so on. This new vocational openness means a structural, conceptual, methodological hybridation. Knowledge, theories, skills traditionally divided among specific fields must combine and melt together as stated in the Manifesto ibridi (Giacoma, Bocchi, Damiano \& Casali 2012) when explaining the trans-disciplinary attitude: "Hybrids are those people, situated in the most various professional, cultural, and scientific pathways, who are able to connect traditionally separated fields of knowledge and action" according to the four pillars of the new design challenge: complexity, acceleration, interaction and mind.

On the other side, design disciplines must converge towards the focus of their own specific conceptual core, contaminating themselves across the several souls and languages.

Therefor design should face with the mutation of culture in itself as well described by Baricco in the essay I barbari —or a hybrid pamphlet published on a daily newspaper before to become a book in 2006 - from a vertical culture - intended as a meaningful diving in the depths of knowledge - to an horizontal one - represented by movement and shifting among superficial experience to another. Despite the skeptical vision of the author, this two axis well descrivbe the attitude that next generation must develop to be able to face the future challenges. A mutation that is atropological.

In less than ten years a new generation of students and then deigners will begin to replace the digital migrant pros. Millenials or digital natives will bring in this world a different point of view. They will be the first generarion both of users and professionals to be born in the digital era. According to the definition given by Perkins (2001) they are identified as a new group born after 1985 grown up with digital technologies such as computers, Internet, mobile phones and MP3s already naturally familiar with digital technologies and devices. As reported by Schmidt and Hawkins (2008) in their recent paper Children of the tech revolution what is changing are the values, the way they interact with the world and how they connect socially to each other. Richard Watson in his Future files: a brief history of the next 50 years (2010) particularly stress the way how their digital attitudes embed and raise soft skills and social values based on multi-play computer gaming, collaboration, leadership, co-operation and problem solving skills.

They are also an information-intensive generation: always connected to internet, accustomed to free access to knowledge sources such as wikipedia, news, freeware software or on line SAAS (software as a service). Generation $\mathrm{Z}$ and Millennials have also a strong attitude to sharing culture and collaborative relation not only in terms of collectivization of emotion and competences thrue the revolution of web 2.0 - just to mention the huge amount of tutorials, peer reviews, free lessons and resources available on Youtube or forums and Q\&A groups produced and distributed both by pros and amateurs - social networks, MOOCs and peer-to-peer platforms.

This will be the cultural and social context in which it would be possible to reform and develop a new, open, hybrid and eco-systemic design culture firm and deeply rooted in its traditional basic principles and at the same time open to the material and technological evolution, not yet foreseeable but already certain.

\section{References}

ANDERSON, J. (2011). "Change on a Dime: Agile Design. An overview of agile development and working as a designer in agile environments." In Ux Magazine, 700, 19 July. $<$ https://uxmag.com/articles/change-on-a-dime-agiledesign> [Visited: 6 May 2016].

BARICCO, A. (2006). I barbari. Saggio sulla mutazione. Feltrinelli: Milano.

BECK, K. et Al. (2001). Manifesto for Agile Software Development. Agile Alliance.

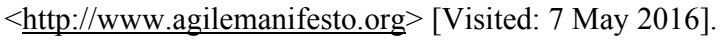


Fixed, liqid, fluid. Rethinking the digital design process through the ecosystem model.

BOLLINI, L. (2004). Regstrica Multimodale. Il design dei new media. Maggioli Editore: Santarcangelo di Romagna.

BONSIEPE, G. (1993). Il ruolo del design. In Anceschi, G. (Ed.). Il progetto delle interface. Oggetti colloquiali e protesi virtuali. Domus Academy: Milano

GIACOMA, G.; BOCCHI, G.; DAMIANO, L. and CASALI, D. (2012). Manifesto ibridi. $<$ http://manifestoibridi.org/explanation/it> [Visited: 7 May 2016].

GUSTAFSON, A. (2008) Understanding Progressive Enhancement, A list Apart 269, 7 October. $<\underline{\text { http://alistapart.com/article/understandingprogressiveenhancement }}>$ [Visited: 7 May 2016].

LEHMAN, M. M. (1969). The Programming Process. IBM Research Report RC 2722, IBM Research Center. Yorktown Heights: New York.

LEVIN, M. (2014). Designing Multi-Device Experiences. An Ecosystem Approach to User Experiences Across Devices. O'Reilly Media: Sebastopol.

MALDONADO, T. (1991). Disegno industrial: un riesame. Feltrinelli: Milano.

MALDONADO, T. (1994). Reale e virtuale. Feltrinelli: Milano.

MATURANA, H. R. \& VARELA, F. J. (1984). Tree of Knowledge: Biological Roots of Human Understanding. Shambhala Publications Inc: Boulder.

MOLINA, B. (2011). Facebook unveils Timeline profiles. In Usatoday 22 September. $\leq$ http://content.usatoday.com/communities/technologylive/post/2011/09/live-coverage-facebooks-f8-developerconference/1\#.UMBu_axp6jY> [Visited: 7 May 2016].

PERKINS, M. (2001). Digital natives, digital immigrants part 1. In On the horizon, 9(5), 1-6.

POLILLO R. (1993). Il design dell'intrazione. In Anceschi, G. (Ed.). Il progetto delle interface. Oggetti colloquiali e protesi virtuali. Domus Academy: Milano.

POPPENDIECK, M. \& POPPENDIECK, T. (2003). Lean Software Development: An Agile Toolkit. Addison-Wesley Professional: Boston.

ROGERS, N. (1952). Dal cucchiaio alla città. In XIII congresso dell'Icsid (International Council of Societies of Industrial Design).

ROSATI, L. (2011). Cross-channel, cross-media, multi-channel: quale differenza? In Architettura dell'informazione e trovabilità. 22 August. $<$ http://lucarosati.it/blog/cross-channel $>$ [Visited: 7 May 2016].

SCHMIDT, T. S. (2006) Inside the Backlash Against Facebook. In Time, 06 September $<$ http://www.time.com/time/nation/article/0,8599,1532225,00.html $>$ [Visited: 7 May 2016].

SCHMIDT, L., \& HAWKINS, P. (2008). Children of the tech revolution. In Sydney Morning Herald.

TAKEUCHI, H. \& NONAKA, I. (1986). New Product development game. In Harvard Business Review. 1 January

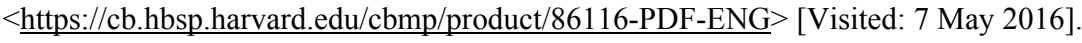

TURKLE, S. (1995). The life on Screen. Identity in the Age of the Internet. Simon \& Schuster: New York.

VERHEYEN, G. (2013). Scrum: framework, not methodology. 21 March. $<$ https://guntherverheyen.com/2013/03/21/scrum-framework-not-methodology > [Visited: 7 May 2016].

VISCO, C. (2012). Mark Zuckerberg: 'Il futuro di Facebook è nel traffico mobile' in Wired, 12 September. $<$ http://daily.wired.it/news/internet/2012/09/12/zuckerberg-tech-crunch-disrupt-intervista-183456.html $>$ [Visited: 7 May 2016].

VERDNASKIJ, V.I. (1926). The Biospehere. [VERDNASKIJ, V.I. (1986). The Biosphere: Complete Annotated Edition. Synergetic Press: Santa Fe].

WATSON, R. (2010). Future files: a brief history of the next 50 years. Nicholas Brealey Publishing.

ZELDMAN, J. (2001). Taking your talent to the web: a guide for the transitioning designer. New Riders: San Francisco. 